\section{Revalidação da escala mobile users' information privacy concerns para o contexto brasileiro}

\author{
Sady Darcy da Silva Junior, Edimara Mezzomo Luciano e Rafael Mendes \\ Lübeck
}

\section{RESUMO}

O objetivo desta pesquisa foi revalidar a escala Mobile Users' Information Privacy Concerns (MUIPC) ao contexto brasileiro, comparando os resultados obtidos aos do contexto norteamericano. Tratou-se de um estudo quantitativo composto por uma amostra de 309 respondentes brasileiros, que responderam via web as questões retrotraduzidas da escala MUIPC. Os dados coletados foram submetidos à análise fatorial exploratória e os resultados obtidos assemelharam-se a uma das pesquisas anteriores realizadas com respondentes norteamericanos. A principal contribuição do estudo foi a adaptação de uma escala de medição da preocupação com a privacidade das informações para usuários de apps de equipamentos mobile para a língua portuguesa, que poderá auxiliar pesquisadores brasileiros em investigações no contexto nacional.

Palavras-chave: preocupação com a privacidade da informação; apps; mobile users information privacy concerns scale.

\section{Revalidation of the Mobile Users' Information Privacy Concerns Scale for the Brazilian context}

\section{ABSTRACT}

The objective of this paper was to revalidate the Mobile Users' Information Privacy Concerns scale (MUIPC) to the Brazilian context, by comparing the results obtained to those of the North American context. The quantitative study was composed of a sample of 309 Brazilian respondents, who answered via web the retro-transcribed questions of the MUIPC scale. Data were analysed to the exploratory factorial analysis and the results obtained resembled one of the previous surveys, with North American respondents. The main contribution of the study was the adaptation of a measurement scale of the concern with the privacy of mobile devices users' information in the Portuguese language, which could help Brazilian researchers in investigations in the national context.

Keywords: information privacy concern; apps; mobile users information privacy concerns scale.
Recebido em: 21/12/2018 Revisado em: 08/02/2019 Aprovado em: 09/04/2019

Check for updates

Sady Darcy da Silva Junior (iD,

Instituto Federal de Educação, Ciência e Tecnologia do Rio Grande do Sul, Brasil

Doutor em Administração, Pontifícia

Universidade Católica do Rio Grande do Sul, Brasil

sady.junior@restinga.ifrs.edu.br

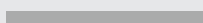

\section{Edimara Mezzomo} Luciano iD,

Pontifícia Universidade Católica do Rio Grande do Sul, Brasil

Doutora em Administração, Universidade Federal do Rio Grande do Sul, Brasil

eluciano@pucrs.br

\section{Rafael Mendes Lübeck (iD,} Pontifícia Universidade Católica do Rio Grande do Sul, Brasil

Doutor em Administração, Pontifícia Universidade Católica do Rio Grande do Sul, Brasil

rafael.lubeck@pucrs.br 


\section{Introdução}

Privacidade de informações, em termos conceituais, é a reivindicação de indivíduos, grupos ou instituições para determinarem quando, como e em que medida as informações sobre si mesmos são comunicadas a outros (Kaushik, Jain, \& Singh, 2018; Westin, 2003; 1967). Em função disso, as pessoas têm uma necessidade de privacidade das informações que, em conjunto com as necessidades de divulgação, tais como a socialização, Ihes ajudam a ajustar-se emocionalmente para a vida diária com os outros (Haans, Kaiser, \& Kort, 2007).

Privacidade das informações também pode ser definida como um mecanismo de controle seletivo do acesso a si próprio ou a um grupo, considerando aspectos sociais e interpessoais (Altman, 1976), ou ainda como a capacidade do indivíduo para controlar pessoalmente as informações sobre si (Stone, Gueutal, Gardner, \& McClure, 1983). O assunto tem voltado à discussão na medida em que mais e mais informações de usuários de dispositivos digitais são coletadas sobre, em grande parte, sua atuação enquanto usuários de dispositivos móveis.

Um dos entendimentos sobre privacidade é que o usuário toma uma decisão deliberada sobre o quanto ele deseja se expor e isso indica o quanto ele se preocupa ou não com a sua privacidade. Entende-se, nesta pesquisa, que esta decisão é socialmente construída, portanto, os usuários podem apresentar diferentes níveis de preocupação com privacidade dependendo de fatores locais e culturais, o que torna importante a validação do instrumento de pesquisa no contexto brasileiro.

Mylonas, Kastania e Gritzalis (2013) analisaram a consciência dos usuários de smartphones em termos de segurança, ao baixarem aplicativos dos repositórios oficiais de apps (por exemplo, Google Play, Apple Store etc.). Como resultado, verificou-se uma complacência, mostrando que a maioria dos usuários confia nos repositórios, não habilitando os controles de segurança no aparelho e até mesmo desprezando a questão da segurança durante a seleção e instalação de apps. Assim, o ato de instalar apps acaba tornando esses repositórios oficiais verdadeiros vetores de ataque à segurança e à privacidade.

Outro ponto que se destaca acerca do tema privacidade das informações diz respeito ao local onde é realizada a coleta de dados (Fodor \& Brem, 2015). Em um estudo que buscava uma análise crítica da literatura, que considera a privacidade de informações como construto chave (Bélanger \& Crossler, 2011), verificou-se que as a maioria das pesquisas foi realizada nos Estados Unidos. Devido haver sido realizada em apenas um contexto cultural (o norte-americano) limita-se a generalização dos resultados. Entretanto, essa concentração de pesquisas nos Estados Unidos cria oportunidades para investigações em outros países.

Para ilustrar essa afirmação estudos referentes à escala MUIPC (Mobile Users' Information Privacy Concerns) basearam suas análises em dados coletados nos EUA, apesar de serem professores da Universidade de Leibniz/ Hannover, na Alemanha (Degirmenci, Guhr \& Breitner, 2013; Xu, Gupta, Rosson, \& Carroll, 2012). Os EUA têm uma forte reputação no contexto das 
pesquisas sobre privacidade das informações. Desta forma, os participantes norte-americanos podem ter crenças fortes e bem formadas sobre o acesso a informações pessoais e preocupações com a privacidade (Degirmenci, Guhr, \& Breitner, 2013).

Já em termos de estruturas reguladoras, vários países têm abordado a questão da privacidade de forma diferenciada (Smith, 2004), além do fato da cultura de um país ter impacto duradouro sobre a forma como se lida com a privacidade (Chang \& Chen, 2008). Assim, recomenda-se que pesquisas futuras sobre a preocupação com a privacidade da informação devam ser conduzidas em outros países (Yun, Lee, \& Kim 2019; Degirmenci, Guhr \& Breitner, 2013), buscando uma amostra diversificada para uma possibilidade de maior generalização da MUIPC (Xu-Gupta et al., 2012). Com base no exposto acima, o objetivo desta pesquisa é comparar os resultados obtidos com a aplicação da versão traduzida da escala MUIPC no contexto brasileiro com aqueles obtidos no contexto norte-americano, buscando identificar semelhanças e/ou diferenças.

\section{Referencial teórico}

Várias abordagens para conceituar a privacidade das informações têm sido propostas na literatura. Em função disso, identificou-se quatro abordagens de definição para a privacidade da informação: privacidade como um direito humano; privacidade como uma mercadoria; privacidade como um estado de acesso limitado e privacidade como a capacidade de controlar a informação sobre si mesmo (Smith, Dinev, \& Xu, 2011; Tang \& Lin, 2017).

Em relação à importância da privacidade das informações, ela já foi citada como uma das mais importantes questões éticas, legais, sociais e políticas da era da informação (Culnan \& Bies, 2003; Mason, 1986; Millberg, Smith, \& Burke, 2000). Além do mais, em função do crescente aumento de informações pessoais no meio digital e dos avanços tecnológicos para internet, surgem cada vez mais novos desafios para se conseguir manter a privacidade das informações dos consumidores (Angst \& Agarwal, 2009; Ward, Bridges \& Chitty, 2005).

Em tais circunstâncias, embora a noção de privacidade própria possa parecer algo simples, o limite prático de privacidade de informação na vida real varia de acordo com inúmeros fatores, incluindo os setores da indústria, culturas e leis reguladoras (Culnan \& Bies, 2003; Milberg, Burke, \& Smith, 1995). Havendo tais particularidades, a preocupação com a privacidade de informações tem contornos mais específicos quando se refere ao indivíduo. Sendo assim, a próxima seção irá discorrer sobre esse tema.

\section{Privacidade do indivíduo}

A preocupação com a privacidade das informações representa a extensão em que um indivíduo está preocupado com as práticas organizacionais relacionadas à coleta e uso de suas informações pessoais (Smith, Milberg, 
\& Burke, 1996). Sendo assim, o foco não é a forma como um indivíduo percebe as políticas de uma organização em relação à privacidade de suas informações, mas, ao invés disso, como percebe as preocupações mais amplas em torno de coleta de informações e uso por organizações em geral (Malhotra, Kim, \& Agarwal, 2004).

As preocupações com as informações pessoais referem-se a visões subjetivas de um indivíduo do conceito de justiça, dentro do contexto da privacidade da informação (Campbell, 1997). Em termos de consequências deste nível de preocupação, verificou-se que a preocupação com a privacidade das informações pode afetar a percepção de invasão de privacidade do consumidor em um contexto específico (Schwaig, Segars, Grover, \& Fiedler, 2013).

As preocupações de um indivíduo com a privacidade serão influenciadas pelas condições externas. No entanto, a percepção de um indivíduo de tais condições externas também irá variar em acordo as características pessoais e experiências passadas (Donaldson \& Dunfee, 1994). Por isso, muitas vezes, as pessoas têm opiniões diferentes sobre o que é justo e o que não é justo em relação à coleta e uso de suas informações pessoais por parte de uma empresa. Entretanto, de qualquer forma os consumidores são vulneráveis nas suas relações com as empresas, devido à falta de informações e à total incapacidade de controlar o uso posterior de suas informações pessoais (Culnan \& Williams, 2009).

Corroborando com esse cenário, pesquisas têm demonstrado que os consumidores on-line estão muito preocupados com os esforços das empresas em proteger seus dados pessoais após terem sido divulgados (Buchanan, Paine, Joinson, \& Reips, 2007; Pavlou, Liang, \& Xue, 2007), sendo fundamental então que as empresas adotem medidas e formulem políticas de proteção à integridade das informações dos clientes (Stewart \& Segars, 2002).

Reforçando essa preocupação, estudos recentes vêm enfatizando a importância da preocupação com a privacidade nas mais diversas situações: a) Quando a internet é utilizada como um meio para a transferência de informações (Hui, Teo, \& Lee, 2007; Dinev \& Hart, 2006; Malhotra, Kim, \& Agarwal, 2004); b) Quando as informações são coletadas e usadas em um contexto organizacional (Hong \& Thong, 2013; Stewart \& Segars, 2002; Smith, Milberg, \& Burke, 1996); c) Analisando a forma como os indivíduos respondem a ameaças à privacidade eletrônica (Son \& Kim, 2008); e d) Quando as informações são coletadas e usadas aplicativos móveis (Degirmenci, Guhr, \& Breitner, 2013; Xu, Gupta et al. 2012).

\section{Escalas de medição da preocupação com a privacidade das informações}

Com o passar dos anos a preocupação com a privacidade na Internet vêm recebendo cada vez mais atenção, devido à quantidade cada vez maior de informações pessoais que estão sendo coletadas, armazenadas, transmitidas e publicadas na Internet. Além disso, embora exista uma literatura emergente sobre o assunto, há um acordo limitado sobre sua 
conceituação em termos de dimensões-chave (Hong \& Thong, 2013). Nessa seção, discute-se três importantes escalas de medição de privacidade de informações. Inicialmente foram descritas as três escalas e ao final discutiu-se as interações entre elas e características que as diferem entre si.

\section{Concerns for Information Privacy Scale}

Criada dentro do contexto do marketing direto off-line, a CFIP - Concerns for Information Privacy - é uma escala que mede as dimensões primárias das preocupações individuais sobre as práticas organizacionais a respeito da privacidade da informação, composta por quatro dimensões (Smith, Milberg, \& Burke, 1996): Coleta, Uso Secundário Não Autorizado, Acesso Indevido e Erros. Posteriormente, a CFIP teve sua confiabilidade validada empiricamente como um modelo de fator de segunda ordem (Stewart \& Segars, 2002).

Dentre as escalas de medição da preocupação dos indivíduos com a privacidade das informações pessoais, destaca-se a CFIP - Concern for Information Privacy (Smith, Milberg, \& Burke, 1996), devido ao pioneirismo no assunto e de ter servido de base para uma série de pesquisas que buscaram readequações e aprimoramentos em relação à sua estrutura original (Bélanger \& Crossler, 2011).

\section{Internet Users' Information Privacy Concerns Scale}

A primeira escala que buscou uma atualização da CFIP foi a IUIPC Internet Users' Information Privacy Concerns, que foi concebida a partir da identificação do problema da falta de confiança dos consumidores com a privacidade das informações, o que dificultava o crescimento do e-commerce na época de sua criação (Malhotra, Kim, \& Agarwal, 2004). Em função disso, foi originalmente desenvolvida para refletir as mudanças na percepção de um indivíduo sobre a equidade e justiça, em função do uso generalizado da Internet.

A IUIPC foi fortemente enraizada na Teoria do Contrato Social (Donaldson \& Dunfee, 1994), que lança luz sobre a natureza das relações de longo prazo entre as partes interessadas. Por esse motivo, tornou-se uma representação eficiente e eficaz das preocupações dos consumidores on-line relativas à privacidade das informações, concentrando-se nas percepções dos indivíduos sobre equidade e justiça, conforme citado anteriormente.

Em termos de estrutura, a IUIPC é um modelo de fator de segunda ordem com três fatores de primeira ordem (Coleta, Controle e Consciência), que foram concebidos a partir da adaptação da escala da CFIP para o contexto da Internet. Como resultado, a IUIPC sugere que a coleta de informações dos indivíduos por parte de uma empresa somente pode ser percebida como justa quando é concedido ao consumidor o controle sobre a informação, além de ficar claro para o mesmo o uso pretendido das informações por parte empresa.

\section{Internet Privacy Concerns Scale}

Diante da evolução dos estudos, foi concebida a IPC - Internet Privacy Concerns, que tem como objetivo estudar as preocupações com a privacidade na internet como uma percepção da relação entre o indivíduo e 
uma entidade online, que pode ser um site específico ou uma categoria de sites, como sites comerciais (Hong \& Thong, 2013). Em termos de estrutura, a IPC é um fator geral de terceira ordem (IPC), com dois fatores de segunda ordem (Gestão da Interação e Gestão da Informação) e seis fatores de primeira ordem elaborados a partir da junção das dimensões da CFIP (Smith, Milberg, \& Burke, 1996) e da IUIPC (Malhotra, Kim, \& Agarwal, 2004): Coleta, Uso Secundário, Erros, Acesso Indevido, Controle e Consciência.

\section{Histórico das escalas}

O primeiro estudo posterior à escala CFIP mostrou que suas quatro dimensões originais (Coleta, Uso Secundário Não Autorizado, Acesso Indevido e Erros) podem ser agrupadas como um fator de segunda ordem (CFIP), que compõem o construto da preocupação com a privacidade das informações (Stewart \& Segars, 2002). O que demonstra a relevância da CFIP é o fato da mesma servir de base aos três estudos subsequentes que compõem a sequência de progresso das principais escalas de preocupação com a privacidade da informação.

Em relação à primeira escala que apresentou-se posteriormente à CFIP, a IUIPC - Internet Users'Information Privacy Concerns (Malhotra, Kim, \& Agarwal, 2004), apesar de normalmente ser citada em pesquisas subsequentes, ela tem sido pouco utilizada, uma vez que a maioria das pesquisas relacionadas às questões de preocupação de privacidade continua a utilizar a escala inicial CFIP (Hong \& Thong, 2013; Bélanger \& Crossler, 2011). Entretanto, chama atenção o primeiro autor do artigo seminal da IUIPC ser Malhotra (2006), autor do renomado livro Pesquisa de marketing: Uma orientação aplicada. Inclusive, no livro cita-se o IUIPC, afirmando que se comprovou que a escala tem boa confiabilidade e validade de construto.

Talvez por esta pouca utilização, a segunda tentativa de atualização da escala CFIP tenha sido sugerida apenas recentemente, através da escala denominada IPC - Internet Privacy Concerns (Hong \& Thong, 2013), a partir justamente de adaptações das estruturas da CFIP e da IUIPC. Essa pesquisa mostra-se relevante pelo fato de ter sido a primeira tentativa robusta de junção das duas escalas anteriores - CFIP e IUIPC.

Similarmente à escala IPC, com base nas escalas CFIP e IUIPC, foi proposta a escala MUIPC - Mobile Users' Information Privacy Concerns, (Xu et al., 2012), voltada ao contexto específico e extremamente atual do uso dos aplicativos móveis. Posteriormente, a MUIPC foi testada avaliando se a preocupação com a privacidade da informação dos usuários de dispositivos móveis era influenciada pelo acesso há alguns tipos de informação pessoal (Degirmenci, Guhr, \& Breitner, 2013). Como resultado, além de ampliar a pesquisa sobre a MUIPC, verificou-se que a preocupação dos usuários é influenciada pelo acesso à identificação pessoal, à localização e ao conteúdo do dispositivo móvel.

Em termos de utilização, a escala CFIP vem sendo amplamente aplicada em diferentes contextos, que vão desde o marketing direto (Smith, Milberg, \& Burke, 1996) e e-commerce (Van Slyke, Craig Shim, Richard, \& Jiang, 2006), até o setor de saúde (Angst \& Agarwal, 2009). Isso demonstra, em parte, a relevância do CFIP, que fica ainda mais evidenciada pelo fato de atualmente 
ainda ser a escala mais utilizada em pesquisas relacionadas às preocupações com a privacidade da informação (Bélanger \& Crossler, 2011; Hong \& Thong, 2013), apesar de ter sido a primeira escala proposta sobre o assunto e com várias escalas propostas a partir dela.

Ao avaliar o histórico das escalas, chama atenção o gap temporal entre as primeiras escalas de medição da preocupação com a privacidade das informações (CFIP/CFIP como fator de Segunda Ordem/IUIPC) e as lançadas nos últimos anos (IPC+MUIPC). Assim, percebe-se uma atualização das mesmas para a realidade vigente, dada a atenção cada vez maior que a questão da preocupação com a privacidade das informações vem recebendo (Hong \& Thong, 2013; Xu et al., 2012). Portanto, isso torna o construto "preocupação com a privacidade das informações" como algo importante para as pesquisas na área de administração de sistemas de informação (Kaushik, Jain, \& Singh 2018; Xu et al. 2011).

\section{MUIPC - Mobile Users' Information Privacy Concerns}

A MUIPC diz respeito às preocupações com a possível perda de privacidade, resultante da divulgação de informações pessoais para um agente externo específico, surgindo a partir da constatação da necessidade de mudança nas dimensões até então existentes referentes às preocupações com a privacidade das informações. Visto que, os usuários de dispositivos móveis são provavelmente diferentes dos consumidores on-line, em termos de percepção das ameaças à privacidade. Sendo assim, quanto à estrutura, a MUIPC caracteriza-se como um modelo de fator de segunda ordem (MUIPC) com três fatores: Vigilância Percebida, Intrusão Percebida e Uso Secundário de Informações Pessoais (Xu et al., 2012).

Outro fator de motivação para a criação da MUIPC foi a verificação da ausência de estudos sobre os riscos de privacidade voltados especificamente para o ambiente que envolve tecnologias de redes móveis e smartphones. Além disso, motivou também o desenvolvimento da MUIPC o fato das questões sobre a privacidade no contexto dos dispositivos móveis tornarem-se extremamente importantes e cada vez mais imprescindíveis, visto que os vendedores de apps podem acessar um volume cada vez maior de informações pessoais, na medida em que aumenta de forma sem precedentes o acesso dos consumidores à Internet e a serviços de valor agregado, a qualquer hora e em qualquer lugar (Xu et al., 2012).

Em termos de escala, cada uma das três dimensões referentes aos fatores de primeira ordem foi construída utilizando como base a Teoria da Gestão de Privacidade na Comunicação (CPM - Communication Privacy Management) (Petronio, 2002), além das escalas CFIP (Smith, Milberg, \& Burke, 1996) e IUIPC (Malhotra, Kim, \& Agarwal, 2004), possuindo três itens cada, totalizando, assim, nove itens. Os significados de cada uma das três dimensões da MUIPC, bem como a base que as gerou, além do CPM, são os seguintes (Xu et al., 2012):

a. Vigilância Percebida: vigilância pode ser definida como a observação, escuta ou gravação das atividades de um indivíduo (Solove, 2006). No ambiente móvel de hoje, os vendedores aproveitam as 
poderosas tecnologias de vigilância para monitorar o perfil dos consumidores, que podem então resistir aos aplicativos móveis por medo de que suas atividades possam ser observadas, registradas e transmitidas para diversas entidades (Xu et al., 2012). Em termos de embasamento, a dimensão da vigilância percebida endereça a dimensão de Coleta do CFIP e do IUIPC;

b. Intrusão Percebida: intrusão pode ser definida como atos invasivos que perturbam a tranquilidade ou a solidão dos indivíduos, envolvendo a incursão geral indesejada, quando na presença ou através das atividades de outros (Solove, 2006). Já a percepção da intrusão por parte dos indivíduos é causada quando os destinatários das informações pessoais dos indivíduos são capazes de tomar decisões independentes a respeito das mesmas (Xu et al., 2012). Em termos de embasamento, a dimensão de intrusão percebida endereça as dimensões de Acesso Inadequado e Erros do CFIP, além da dimensão de Controle do IUIPC;

c. Uso Secundário de Informações Pessoais: da mesma forma que no CFIP, significa as situações em que as informações pessoais dos indivíduos são coletadas para uma finalidade, porém são usadas para outra, sem a autorização dos mesmos (Smith, Milberg, \& Burke, 1996). Como exemplo, tem-se perfil dos indivíduos e envio de mensagens de marketing. Em termos práticos, o potencial para uso secundário gera medo e incerteza sobre a forma como as informações pessoais serão usadas no futuro, criando uma sensação de impotência e vulnerabilidade (Solove, 2006). Quanto ao embasamento, endereça diretamente a dimensão de Uso Secundário Não Autorizado do CFIP.

Considerando o cenário descrito, demonstrou-se a evolução dos estudos quantitativos sobre privacidade de informações. Evidencia-se a importância da escala CFIP como base para os estudos anteriores, mas ressalta-se que, ao integrar ou rever as dimensões de privacidade de informações, as escalas posteriores à CFIP representam um ajuste mais adequado ao cenário presente. Tal ajuste se materializa quando se entende que as transformações geradas pela tecnologia criam constantemente novos desafios, possibilidades e afetam a privacidade de informações devido ao crescimento da quantidade, precisão das informações e dos mecanismos que as geram, independente da vontade dos indivíduos.

Cabe, então, revisitar os conceitos originais e ajustá-los com o passar do tempo. Assim, a escala analisada nesse estudo (MUIPC) representa atualização do conhecimento sobre privacidade de informações. Por fim, ressalta-se que a adaptação de escalas a determinados contextos é importante não apenas para sua aplicação e ampliação do conhecimento local, mas também é uma forma de ampliar o alcance da escala rumo às teorias mais consistentes globalmente. 
Método

Esta pesquisa é quantitativa e, para facilitar o entendimento das etapas desse estudo, foi criado um desenho de pesquisa que ilustra as atividades que foram realizadas visando à execução do trabalho (Quadro 1).

Quadro 1: Desenho da pesquisa

\begin{tabular}{ll}
\hline Etapa 1 & $\begin{array}{l}\text { Questão de pesquisa: os resultados da escala MUIPC traduzida e aplicada ao contexto brasileiro, considerando fatores e } \\
\text { variáveis, serão semelhantes àqueles obtidos pelos estudos anteriores com respondentes norte-americanos? }\end{array}$ \\
Etapa 2: & $\begin{array}{l}\text { Objetivo da pesquisa: comparar os resultados obtidos com a aplicação da versão traduzida da escala MUIPC no contexto } \\
\text { brasileiro em relação ao contexto norte-americano, buscando identificar semelhanças e diferenças. }\end{array}$ \\
Etapa 3 & $\begin{array}{l}\text { Referencial teórico: Preocupação com a Privacidade de Informações/Escalas de medição da preocupação com a } \\
\text { privacidade das informações/MUIPC - Mobile Users'Information Privacy Concerns. }\end{array}$ \\
Etapa 4: & $\begin{array}{l}\text { Preparação para a coleta: retrotradução da escala MUIPC/cadastro em aplicativo web apropriado para surveys on-line. } \\
\text { Etapa 5: }\end{array} \quad \begin{array}{l}\text { Coleta de dados: disponibilização de link de acesso para rede de contato dos pesquisadores e do Facebook (período de } \\
\text { Etapa 6 }\end{array} \quad \begin{array}{l}\text { Análise de dados: Alfa de Cronbach/Teste de KMO e Bartlet/Análise Fatorial Exploratória. } \\
\text { Etapa 7: }\end{array} \quad \begin{array}{l}\text { Conclusões e considerações finais: conclusões dos resultados obtidos, cruzando-0s com a questão de pesquisa e } 0 \\
\text { respectivo objetivo e por fim limitações da pesquisa e sugestões de estudos futuros. }\end{array}$ \\
\hline
\end{tabular}

Fonte: Elaborado pelos autores (2019)

Inicialmente, foi necessária a tradução da escala original ( $X u$ et al., 2012) para a língua portuguesa. Para evitar que a tradução direta de certas palavras e frases transcorresse de forma incorreta, foi utilizada a técnica de retrotradução (Malhotra, 2006). Após esse procedimento, a escala MUIPC traduzida foi cadastrada em um aplicativo web, para que fosse possível sua disponibilização através de um link de acesso, sendo divulgada por meio da rede de contato dos pesquisadores e de redes sociais.

O aplicativo garantiu que apenas uma alternativa fosse selecionada em cada uma das nove questões, bem como que nenhum item ficasse sem ser respondido, impossibilitando o respondente de passar para a próxima. As respostas foram coletadas seguindo o mesmo padrão da escala MUIPC original, via escala de 7 pontos, variando de 1 - Discordo Totalmente até 7 Concordo Totalmente. Foram coletadas 309 respostas válidas obtidas entre setembro e outubro de 2017. Adicionalmente, as estatísticas descritivas e características da amostra foram dispostas nos apêndices $E$ e $F$ para futuras consultas.

As técnicas estatísticas de análise aplicadas aos dados coletados nesta pesquisa, visando à validação da confiabilidade do instrumento de pesquisa e a comparação de sua estrutura traduzida para a língua portuguesa com a estrutura original da MUIPC, bem como uma possível redução inicial, seguiram as recomendações de Hair, Black, Babin, Anderson e Tatham (2009) e Malhotra (2006). As recomendações foram: a) Alfa de Cronbach: Trata-se de um dos testes mais usados para verificar a coerência interna de um conjunto de variáveis, determinando, assim, a confiabilidade de uma medida; b) Testes de KMO e Bartlett: indicam o grau de suscetibilidade ou o ajuste dos dados à análise fatorial, indicando o nível de confiança que pode 
ser obtido dos dados quando tratados pelo método multivariado de análise fatorial; c) Análise Fatorial Exploratória: Análise multivariada das diferentes variáveis, visando verificar a estrutura dos fatores que compõem as escalas, com uso da análise de componente principal e rotação Varimax.

O uso da análise fatorial exploratória ao invés da confirmatória ocorreu porque a escala em estudo, a MUIPC, foi analisada anteriormente em apenas dois estudos e com respondentes norte-americanos, sendo que 0 segundo estudo apresentou uma diferença de resultados, o que ocasionou a eliminação de uma variável. Assim, visto que se trata de uma escala muito recente, com apresentação de resultados diversos, retrotraduzida para o idioma português e aplicada com respondentes brasileiros, optou-se pela análise fatorial exploratória. Além disso, o objetivo desta pesquisa é uma comparação dos resultados dos estudos, buscando identificação de semelhanças e/ou diferenças e não a validação ou confirmação da escala MUIPC original.

\section{Resultados e discussão}

As análises foram obtidas via SPSS 20 e a confiabilidade da versão traduzida para língua portuguesa da escala MUICP iniciou-se através da análise do Alfa de Cronbach que, segundo Kline (2011), mede a confiabilidade interna e o grau de consistência das respostas entre os itens dentro de uma medida. Foi verificado inicialmente para os 9 itens Alfa de Cronbach 0,900.

O resultado do coeficiente Alfa de Cronbach demonstra a consistência interna da escala MUIPC traduzida, visto que valores acima de 0,60 indicam confiabilidade satisfatória de consistência interna (Malhotra, 2006). Desta forma, considerando-se apenas o índice do coeficiente Alfa de Cronbach obtido, presume-se que não seria necessário retirar questões do instrumento.

Dando prosseguimento à análise, foi realizado o Teste de KMO e Bartlett, que representa o grau de suscetibilidade ou o ajuste dos dados à análise fatorial, indicando o nível de confiança que pode ser obtido com os dados quando tratados pelo método multivariado de análise fatorial (Hair et al., 2009). De acordo com Malhotra (2006), valores do índice de KMO inferiores a 0,5 indicariam que a análise fatorial poderia ser inadequada. Já o teste de esfericidade serve para validar a utilização da análise fatorial, que ocorre quando a significância é inferior a ,050. Assim, fica claro que os índices obtidos foram consistentes: $\mathrm{KMO}$ - 0,900; $\mathrm{x}^{2}=1.493,416$; $\mathrm{GL} 36$; $\mathrm{P}<0,001$.

A validação seguinte foi a análise fatorial exploratória (AFE), com o objetivo de verificar a estrutura dos fatores que compõem a escala, utilizando Análise de Componentes Principais e Rotação Varimax (Hair et al., 2009). A primeira avaliação oriunda da AFE foi a comunalidade das variáveis que, de acordo com Malhotra (2006), é a porção da variância que uma variável compartilha com todas as outras variáveis consideradas, também interpretada como a proporção de variância explicada pelos fatores comuns.

Para fins de interpretação, valores de comunalidade abaixo de 0,5 indicam que a variável não fornece explicação suficiente para o que está mensurando, sendo necessária uma amostra maior. A maioria dos valores 
obtidos são satisfatórios $(>0,50)$ e somente VP1 ficou abaixo do indicado (ver Tabela 1), enquanto a Variância Total Explicada foi de 57,046\%.

Por fim, foram avaliadas as cargas fatoriais constantes na matriz de fatores, a fim de se observar o grau de correlação entre os fatores e as variáveis (ver Tabela 1). Um coeficiente de correlação com valor absoluto grande indica que o fator e a variável estão estreitamente relacionados (Malhotra, 2006). Desta forma, percebe-se mais uma vez um desajuste da variável VP1 em relação ao modelo, em função do baixo grau de correlação com o fator se comparada ao índice de correlação das oito demais variáveis. Neste sentido, é fundamental salientar que esta mesma variável também foi a única a ser eliminada no segundo artigo que abordou a MUIPC (Degirmenci, Guhr, \& Breitner, 2013) após a análise fatorial exploratória realizada sobre dados obtidos com respondentes norte-americanos.

Tabela 1. AFE - 1.a Análise

\begin{tabular}{cccc}
\hline Fator/Dimensão & Variável & Comunalidade & Carga Fatorial \\
\hline \multirow{2}{*}{ Vigilância Percebida } & VP1 &, 189 &, 435 \\
& VP2 &, 739 &, 860 \\
& VP3 &, 614 &, 783 \\
& IP1 &, 551 &, 743 \\
Intrusão Percebida & IP2 &, 570 &, 755 \\
& IP3 &, 559 &, 748 \\
Uso Secundário das & US1 &, 659 &, 812 \\
Informaç̃oes Pessoais & US2 &, 637 &, 798 \\
& US3 &, 616 &, 785 \\
\hline
\end{tabular}

Fonte: Elaborado pelos autores

A partir dessa primeira análise realizada, deixando que o SPSS definisse o agrupamento fatorial das nove variáveis, que resultou em apenas um único fator, partiu-se para uma segunda análise fatorial exploratória, porém realizando as seguintes mudanças: a) Exclusão da variável VP1 do modelo: isso é recomendado em função do baixo índice de comunalidade da mesma e do baixo grau de correlação que apresentou em relação às demais variáveis analisadas; b) Fixação do número de fatores em três: isso foi necessário para uma avaliação mais próxima da escala original MUIPC, uma vez que a mesma apresenta três dimensões, conforme demonstrado anteriormente. Além disso, esse procedimento pode ser realizado porque uma das formas de determinação do número de fatores para realização da AFE é "A Priori", que ocorre quando se tem conhecimento prévio do número de fatores a ser utilizado, podendo também se definir com base na porcentagem de variância, uma vez que deve se obter, no mínimo, 60\% de variância (Malhotra, 2006). Nesse aspecto, o percentual de variância obtido com apenas um fator foi de 57,046\%, conforme demonstrado na 1. análise, abaixo do valor minimamente aceitável.

Iniciou-se então a 2a análise, obtendo o Alfa de Cronbach geral, sem a variável VP1, 0,912; KMO - 0,917; $x^{2}=1.434,749 ; G L 28 ; P<0,001$ e variância total explicada 78,252\%. Observaram-se melhorias dos indicadores 
em relação ao que foi obtido na 1? análise, com as nove variáveis que indicam melhor adequação das variáveis. A seguir, a análise com três fatores foi realizada e comunalidades, cargas fatoriais e alfas de Cronbach foram avaliados (ver Tabela 2).

Tabela 2 - Instrumento final MUIPC adaptado à Língua Portuguesa

\begin{tabular}{|c|c|c|c|c|c|}
\hline Dimensão & Código & Item & Comunalidades & $\begin{array}{l}\text { Cargas } \\
\text { fatoriais }\end{array}$ & $\begin{array}{l}\text { Alfa de } \\
\text { Cronbach }\end{array}$ \\
\hline \multirow{3}{*}{$\begin{array}{l}\text { Vigilância } \\
\text { Percebida }\end{array}$} & VP1 & $\begin{array}{l}\text { Acredito que a localização do meu dispositivo móvel é } \\
\text { monitorada por pelo menos parte do tempo. }\end{array}$ & & Excluída & \multirow{3}{*}{,839 } \\
\hline & VP2 & $\begin{array}{l}\text { Fico preocupado que os apps estejam coletando muitas } \\
\text { informações sobre mim. }\end{array}$ & ,829 & ,659 & \\
\hline & VP3 & $\begin{array}{l}\text { Fico preocupado que os apps possam monitorar minhas } \\
\text { atividades no meu dispositivo móvel. }\end{array}$ &, 904 & ,857 & \\
\hline \multirow{3}{*}{$\begin{array}{l}\text { Intrusão } \\
\text { Percebida }\end{array}$} & IP1 & $\begin{array}{l}\text { Eu sinto que, como resultado do uso de apps, outras } \\
\text { pessoas sabem mais sobre mim do que me é confortável. }\end{array}$ & ,778 &, 777 & \multirow{3}{*}{,806 } \\
\hline & IP2 & $\begin{array}{l}\text { Eu acredito que, como resultado do uso de apps, } \\
\text { informações sobre mim que eu considero privadas estão, } \\
\text { agora, mais disponíveis do que eu gostaria para outras } \\
\text { pessoas. }\end{array}$ & ,737 & ,741 & \\
\hline & IP3 & $\begin{array}{l}\text { Eu sinto que, como resultado do uso de apps, informações } \\
\text { a meu respeito estão acessíveis e, se usadas, minha } \\
\text { privacidade será invadida. }\end{array}$ & ,720 & ,764 & \\
\hline \multirow{3}{*}{$\begin{array}{l}\text { Uso Secundário } \\
\text { das Informações } \\
\text { Pessoais }\end{array}$} & US1 & $\begin{array}{l}\text { Fico preocupado que os apps possam utilizar minhas } \\
\text { informações pessoais para outras finalidades sem me } \\
\text { notificar ou ter minha autorização. }\end{array}$ &, 784 & ,791 & \\
\hline & US2 & $\begin{array}{l}\text { Quando eu forneço informações pessoais para usar apps, } \\
\text { eu fico preocupado que esses aplicativos possam usar } \\
\text { minhas informações para outras finalidades. }\end{array}$ &, 731 & ,737 & \\
\hline & US3 & $\begin{array}{l}\text { Fico preocupado que apps possam compartilhar minhas } \\
\text { informações pessoais com outras entidades sem obter } \\
\text { minha autorização. }\end{array}$ & ,778 & ,806 & ,852 \\
\hline
\end{tabular}

Fonte: Elaborado pelos autores

Como resultado, os valores obtidos demonstraram-se melhor ajustados, constituindo, desse modo, a estrutura fatorial final desse estudo. Adicionalmente calculou-se no Smart PLS o modelo de $2^{\circ}$. ordem referente à escala e seguindo as orientações de Becker, Klein e Wetzels (2012) e Wong (2016). Os dados corroboram os resultados e foram dispostos no apêndice.

\section{Discussão}

Quando se trata da criação e uso de escalas é recorrente a sugestão de pesquisa futura à aplicação de uma escala em outros contextos, como forma de buscar ajustes e futuras generalizações (ex: Dogruel, Joeckel, \& Vitak 2017; Degirmenci, Guhr, \& Breitner 2013). Entretanto, ao realizar ajustes, a partir de técnicas estatísticas, nem sempre é claro porque certas variáveis e escalas não se adaptam a determinados contextos. 
Várias situações podem trazer dificuldades para a replicação de escalas, mas o fator mais importante é o cultural-legal-social. Isso ocorre especialmente em países emergentes nos quais os níveis de educação e a capacidade de compreensão das questões podem estar abaixo do desejável (Burgess \& Steenkamp 2006). Tal questão aparece no presente estudo e pode ser uma das causas da eliminação de uma variável e da dificuldade de agrupamento nos fatores originais. Amostras em países mais heterogêneos com o Brasil (Burgess \& Steenkamp 2006), ou mesmo o observado no estudo de Degirmenci, Guhr e Breitner (2013), podem necessitar desses ajustes; o que não significa que as escalas percam validade. Os ajustes significam apenas que para o contexto proposto e estudado não houve uma perfeita adequação em relação ao estudo original.

Assim, os resultados dessa pesquisa assemelham-se à pesquisa de Degirmenci, Guhr e Breitner (2013), uma vez que a variável VP1 foi eliminada em ambos os casos. A diferença é que na pesquisa de 2013 não foi necessária a fixação do número de fatores em três, visto que as cargas fatoriais ficaram evidentes com três fatores já na 1․ análise realizada. Entretanto, nos dois estudos, ocorreu a eliminação de uma variável. Tomando como base os resultados alcançados, percebe-se algumas diferenças e semelhanças entre os dois estudos anteriores com a MUIPC e a presente pesquisa. Por exemplo, nos três estudos, tanto em termos de Alfa de Cronbach quanto de cargas fatoriais das variáveis, a sequência das melhores dimensões foi a seguinte: 1.) Uso Secundário de Informações pessoais; $2^{a}$.) Intrusão Percebida e 3.a) Vigilância Percebida.

Outro ponto importante refere-se à variável excluída neste estudo (VP1), que foi a mesma excluída em um dos estudos anteriores sobre a MUIPC: Acredito que a localização do meu dispositivo móvel é monitorada por pelo menos parte do tempo. Isso sugere que tanto os respondentes brasileiros quanto os norte-americanos não possuem um claro entendimento sobre o que seja o monitoramento da localização através do dispositivo móvel, o que é preocupante, uma vez que o bloqueio dessa função é crucial para a privacidade do indivíduo e a permissão à mesma é comumente solicitada no momento de instalação de apps.

Em um estudo realizado no Brasil por Britto-Da-Silva, Luciano e Magnagnagno (2015), utilizando outra escala de preocupação com a privacidade das informações, obteve-se como resultado que as dimensões Uso Secundário (que se assemelha à dimensão Uso Secundário das Informações Pessoais desta pesquisa) e Acesso Indevido (que se assemelha à dimensão Intrusão Percebida desta pesquisa) foram as dimensões que apresentaram maiores índices de preocupação, de um total de seis dimensões avaliadas. Outra pesquisa (Mansouri-Rad, Mahamood, \& Luciano, 2014), sobre preocupação com privacidade em dados de saúde com respondentes brasileiros, americanos e japoneses indicou a baixa preocupação com a privacidade por parte dos respondentes brasileiros.

Desta forma, esses resultados podem ajudar a entender porque a escala MUIPC traduzida para a língua portuguesa e aplicada com respondentes brasileiros tenha sido estatisticamente melhor ajustada especialmente nessas duas dimensões. Além disso, por exclusão e tomando por base esses dois 
estudos, fica uma questão para reflexão: os usuários brasileiros possuem entendimento claro do que seja vigilância ou quais são seus danos, visto que a dimensão Vigilância Percebida foi a que menos se ajustou no contexto brasileiro?

Essa é uma questão preocupante, em virtude dos sofisticados processos de vigilância, que são passíveis de ser estabelecidos à medida em que milhares de informações são coletadas dos dispositivos móveis diariamente, seja pelo uso de apps, redes sociais ou pelo simples deslocamento do indivíduo, via GPS dos dispositivos móveis.

\section{Considerações finais}

A preocupação com a privacidade das informações é um tema cada vez mais recorrente não só em pesquisas acadêmicas, mas também no dia a dia das pessoas. Entretanto, o histórico de escalas de medição propostas referentes ao tema predominantemente são em língua inglesa e aplicadas no contexto norte-americano, no qual a preocupação com privacidade é mais presente entre os usuários de dispositivos móveis. A contribuição dessa pesquisa foi adaptar uma escala de medição da preocupação com a privacidade das informações para língua portuguesa.

Com a segunda análise realizada, verificou-se que as dimensões Uso Secundário das Informações Pessoais e Intrusão Percebida apresentaram resultados similares aos dois estudos anteriores, enquanto a dimensão Vigilância Percebida apresentou diferença nos resultados. Apesar de ter sido sugerida a exclusão da mesma variável que o estudo posterior ao seminal da MUIPC, e das duas questões restantes terem se agrupado no mesmo fator, uma das variáveis apresentou correlação superior a 0,5 em dois fatores. Desta forma, a decisão tomada baseou-se, no que sugere a literatura, no sentido de associar a variável ao fator de maior carga fatorial.

Como continuidade desta pesquisa, sugere-se que a escala proposta seja aplicada de forma composta com outras áreas de conhecimento, tais como: a) cultura nacional, a fim de verificar se as especificidades culturais brasileiras podem impactar no nível de preocupação com a privacidade; b) big data e Data Science, para compreender se as pessoas sentem-se ameaçadas com o crescimento da criação de grandes bases de dados e com o estabelecimento da disciplina de analytics; e c) governança da informação, para entender a percepção das pessoas no momento de definição de políticas de segurança, em comparação ao nível de preocupação com a privacidade da informação.

Por fim, é importante a realização de estudos qualitativos que avaliem algumas inferências obtidas a respeito do usuário brasileiro não ter um claro entendimento das diferenças entre Vigilância Percebida, Intrusão Percebida e Uso Secundário das Informações Pessoais, bem como se possuem entendimento claro do que seja vigilância, que foi a dimensão que apresentou os maiores problemas em termos de ajustes de dados. 


\section{Referências}

Altman, I. (1976). Privacy: a conceptual analysis. Environment and Behavior, 8(1), 7-29.

Angst, C. M., \& Agarwal, R. (2009, junho). Adoption of electronic health records in the presence of privacy concerns: the elaboration likelihood model and individual persuasion, MIS Quarterly, 33(2), 339-370.

Becker, J. M., Klein, K., \& Wetzels, M. (2012). Hierarchical latent variable models in PLS-SEM: guidelines for using reflective-formative type models. Long Range Planning, 45(5), 359-394.

Bélanger, F., \& Crossler, R. E. (2011, dezembro). Privacy in the digital age: a review of information privacy research in information systems. MIS Quarterly, 35(4), 1017-1041

Britto-Da-Silva, V. R., Luciano, E. M., \& Magnagnagno, O. A. (2015). Preocupação com a privacidade na internet: uma pesquisa exploratória no cenário brasileiro. Anais do Encontro de Administração da Informação, Brasília-DF, Brasil, 5.

Buchanan, T., Paine, C., Joinson, A. N., \& Reips, U. D. (2007). Development of measures of online privacy concern and protection for use on the internet. Journal of the American Society for Information Science and Technology, 58(2), 157-165.

Burgess, M. S., \& Steenkamp, J. E.M. (2006). Marketing renaissance: how research in emerging markets advances marketing science and practice. International Journal of Research in Marketing, 23(4), 337-356. DOl: https://doi.org/10.1016/ j.jresmar.2006.08.001.

Campbell, A. J. (1997). Relationship marketing in consumer markets: a comparison of managerial and consumer attitudes about information privacy. Journal of Interactive Marketing, 11(3), 44-57.

Chang H., \& Chen S. (2008). The impact of online store environment cues on purchase intention: trust and perceived risk as a mediator. Online Information Review, 32(6), 818-841.

Culnan, M. J., \& Bies, R. J. (2003). Consumer privacy: balancing economic and justice considerations. Journal of Social Issues, 59(2), 323-342.

Culnan, M. J., \& Williams, C. C. (2009). How ethics can enhance organizational privacy: lessons from the choice point and tjx data breaches. MIS Quarterly, 33(4), 673-687.

Degirmenci, K., Guhr, N., \& Breitner, M. H. (2013, December). Mobile applications and access to personal information: a discussion of users' privacy concerns. International Conference on Information Systems (ICIS). Milan, Italy, 34.

Dinev, T., \& Hart, P. (2006). An extended privacy calculus model for e-commerce transactions. Information System Research, 17(1), 61-80.

Dogruel, L., Joeckel, S., \& Vitak, J. (2017). The valuation of privacy premium features for smartphone apps: the influence of defaults and expert recommendations. Computers in Human Behavior, 77, 230-239. DOl: https://doi.org/10.1016/ j.chb.2017.08.035.

Donaldson, T., \& Dunfee, T. W. (1994). Towards a unified conception of business ethics: integrative social contracts theory. Academy of Management Review, 19(2), 252-284. 
Fodor, M., \& Brem, A. (2015). Do privacy concerns matter for Millennials? Results from an empirical analysis of Location-Based Services adoption in Germany. Computers in Human Behavior, 53, 344-353. DOI: https://doi.org/10.1016/j.chb.2015.06.048

Haans, A., Kaiser, F. G., \& Kort, Y. A. W. D. (2007). Privacy needs in office environments: development of two behavior-based scales. European Psychologist, 12(2), 93-102.

Hair Jr., J. F., Black, W.C., Babin, B. J., Anderson, R. E., \& Tatham, R. L. (2009). Análise Multivariada de Dados (6a ed.). Porto Alegre: Bookman.

Hong, W., \& Thong, J. Y. L. (2013, março). Internet privacy concerns: an integrated conceptualization and four empirical studies, MIS Quarterly, 37(1), 275-298.

Hui, K. L., Teo, H. H., \& Lee, S.Y. T. (2007). The Value of Privacy Assurance: An Exploratory Field Experiment. MIS Quarterly, 31(1), 19-33.

Kaushik, K., Jain, N. K., \& Singh, K. A. (2018, November-December). Antecedents and outcomes of information privacy concerns: role of subjective norm and social presence. Electronic Commerce Research and Applications, 32, 57-68. DOI: https:// doi.org/10.1016/j.elerap.2018.11.003

Kline, R. B. (2011). Principals and Practice of Structural Equation Modeling (3a ed.). New York: Guilford.

Malhotra, N. K. (2006). Pesquisa de Marketing: Uma Orientação Aplicada (4a ed.). Porto Alegre: Bookman.

Malhotra, N. K., Kim, S. S., \& Agarwal, J. (2004, dezembro). Internet Users' Information Privacy Concerns (IUIPC): the construct, the scale, and a causal model. Information Systems Research, 15(4), 336-355.

Mansouri-Rad, P., Luciano, E. M., \& Mahmood. M. A. (2014, November). Telemedicine Adoption issues in the U.S.A. and Brazil: Perception of Healthcare Professionals. 45th Annual Meeting of the Decision Sciences Institute, 2014, Tampa, Florida, USA, 45.

Mason, R. O. (1986, março). Four ethical issues of the information age. MIS Quarterly, 10(1), 4-12.

Milberg, S. J., Burke, S. J. H. J., \& Smith, E. A. K. (1995, December). Values, personal information privacy concerns, and regulatory approaches. Communications of the ACM, 38(12), 65-74.

Milberg, S. J., Smith, H. J., \& Burke, S. J. (2000). Information Privacy: Corporate Management and National Regulation. Organization Science, 11(1), 35-57.

Mylonas, A., Kastania, A., \& Gritzalis, D. (2013). Delegate the smartphone user? Security awareness in smartphone platforms. Computers \& Security, 34, 47-66.

Pavlou, P. A., Liang, H., \& Xue, Y. (2007). Understanding and mitigating uncertainty in online environments: an agency theory perspective. MIS Quarterly, 31(1), 105-136.

Petronio, S. (2002). Boundaries of Privacy: Dialectics of Disclosure. Albany: State University of New York Press.

Pinsonneault, A., \& Kraemer, K. L. (1993). Survey research methodology in management information systems: an assessment. Journal of Management Information Systems, 10(2), 75-105.

Schwaig, K. S., Segars, A. H., Grover, V., \& Fiedler, K. D. (2013). A model of consumers' perceptions of the invasion of information privacy. Information \& Management, 50, $1-12$. 
Smith, H. J. (2004). Information privacy and its management. MIS Quarterly Executive, 3(4), 201-213.

Smith, H. J., Dinev, T., \& Xu, H. (2011). Information privacy research: an interdisciplinary review. MIS Quarterly, 35(4), 989-1015.

Smith, H. J., Milberg, S. J., \& Burke, S. J. (1996). Information privacy: measuring individuals' concerns about organizational practices. MIS Quarterly, 20(2), 167-196.

Solove, D. J. (2006). A taxonomy of privacy. University of Pennsylvania Law Review, 154(3), 477-560.

Son, J. L., \& Kim, S. S. (2008). Internet users' information privacy-protective responses: a taxonomy and a nomological model. MIS Quarterly, 32(3), 503-529.

Stewart, K. A., \& Segars, A. H. (2002). An empirical examination of the concern for information privacy instrument. Information Systems Research, 13(1), 36-49.

Stone, E. F. A., Gueutal, H. G., Gardner, D. G., \& McClure, S. (1983). Field experiment comparing information-privacy values, beliefs, and attitudes across several types of organizations. Journal of Applied Psychology, 68(3), 459-468.

Tang, J. H., \& Lin, Y. J. (2017). Websites, data types and information privacy concerns: a contingency model. Telematics and Informatics, 34(7), 1274-1284. DOI: https:// doi.org/10.1016/j.tele.2017.05.012

Van Slyke, C., Craig Shim, J. T., Richard, J., \& Jiang, J. J. (2006). Concern for information privacy and online consumer purchasing. Journal of the Association for Information Systems, 7(6), 415-444.

Ward, S., Bridges, K., \& Chitty, B. (2005). Do incentives matter? An examination of on-line privacy concerns and willingness to provide personal and financial information. Journal of Marketing Communications, 11(1), 21-40.

Westin, A. F. (1967). Privacy and Freedom. New York: Atheneum.

Westin, A. F. (2003). Social and political dimensions of privacy. Journal of Social Issues, 59(2), 431-453.

Wong, K. K. K. (2016). Mediation analysis, categorical moderation analysis, and higher-order constructs modeling in Partial Least Squares Structural Equation Modeling (PLS-SEM): A B2B Example using Smart Pls. The Marketing Bulletin, 26, $1-22$.

Xu, H., Dinev, T., Smith, J., \& Hart, P. (2011). Information privacy concerns: linking individual perceptions with institutional privacy assurances. Journal of the Association for Information Systems, 12(12), 798-824.

Xu, H., Gupta, S., Rosson, M. B., \& Carroll, J. M. (2012). Measuring mobile users' concerns for information privacy. Thirty-Third International Conference on Information Systems (ICIS), Orlando, Florida, United States, [s.n.].

Yun, H., Lee, G., \& Kim, D. J. (2019). A chronological review of empirical research on personal information privacy concerns: an analysis of contexts and research constructs. Information \& Management, 56(4), 570-601. 
Apêndice A - Escala MUIPC original

\begin{tabular}{ccl}
\hline Dimensão & Código & Item \\
\hline $\begin{array}{c}\text { Perceived } \\
\text { Surveillance }\end{array}$ & VP1 & I believe that the location of my mobile device is monitored at least part of the time. \\
& VP3 & $\begin{array}{l}\text { I am concerned that mobile apps are collecting too much information about me. } \\
\text { I am concerned that mobile apps may monitor my activities on my mobile device. }\end{array}$ \\
\hline $\begin{array}{c}\text { Perceived } \\
\text { Intrusion }\end{array}$ & IP1 & $\begin{array}{l}\text { I feel that as a result of my using mobile apps, others know about me more than I am comfortable with. } \\
\text { I believe that as a result of my using mobile apps, information about me that I consider private is now more } \\
\text { readily available to others than I would want. } \\
\text { I feel that as a result of my using mobile apps, information about me is out there that, if used, will invade } \\
\text { my privacy. }\end{array}$ \\
$\begin{array}{c}\text { Secondary } \\
\text { Use of Personal } \\
\text { Information }\end{array}$ & US1 & $\begin{array}{l}\text { I am concerned that mobile apps may use my personal information for other purposes without notifying } \\
\text { me or getting my authorization. } \\
\text { When I give personal information to use mobile apps, I am concerned that apps may use my information } \\
\text { for other purposes. } \\
\text { I am concerned that mobile apps may share my personal information with other entities without getting } \\
\text { my authorization. }\end{array}$ \\
\hline
\end{tabular}

Fonte: Adaptado de Xu et al. (2012)

\section{Apêndice B - Variância média extraída e confiabilidade composta}

\begin{tabular}{|c|c|c|c|c|c|c|}
\hline Dimensão & Variável & $\begin{array}{c}\begin{array}{c}\text { Other } \\
\text { Loadings }\end{array} \\
\end{array}$ & AVE & CC & $\mathbf{Q}^{2}$ & $f^{2}$ \\
\hline \multicolumn{2}{|c|}{ MUIPC (2․ ordem) } & na & 0,866 & 0,916 & 0,461 & 0,46 \\
\hline \multirow{3}{*}{$\begin{array}{l}\text { Perceived } \\
\text { Surveillance }\end{array}$} & VP1 & - & - & - & - & - \\
\hline & VP2 & 0,81 & \multirow{2}{*}{0,806} & \multirow{2}{*}{0,89} & \multirow{2}{*}{0,644} & \multirow{2}{*}{0,371} \\
\hline & VP3 & 0,788 & & & & \\
\hline \multirow{3}{*}{$\begin{array}{l}\text { Perceived } \\
\text { Intrusion }\end{array}$} & IP1 & 0,695 & \multirow{3}{*}{0,688} & \multirow{3}{*}{0,868} & \multirow{3}{*}{0,524} & \multirow{3}{*}{0,678} \\
\hline & IP2 & 0,688 & & & & \\
\hline & IP3 & 0,758 & & & & \\
\hline \multirow{3}{*}{$\begin{array}{l}\text { Secondary Use } \\
\text { of Personal } \\
\text { Information }\end{array}$} & US1 & 0,796 & \multirow{3}{*}{0,753} & \multirow{3}{*}{0,901} & \multirow{3}{*}{0,625} & \multirow{3}{*}{0,485} \\
\hline & US2 & 0,786 & & & & \\
\hline & US3 & 0,775 & & & & \\
\hline
\end{tabular}

\section{Apêndice C - Validade convergente e discriminante}

\begin{tabular}{ccccc}
\hline & MUIPC & IP & US & VP \\
\hline MUIPC & $\mathbf{0 , 9 3 1}$ & & & \\
IP & 0,863 & $\mathbf{0 , 8 3 0}$ & & \\
US & 0,905 & 0,628 & $\mathbf{0 , 8 6 8}$ & $\mathbf{0 , 8 9 8}$ \\
VP & 0,890 & 0,770 & 0,742 & \\
\hline
\end{tabular}


Apêndice D - Ajustes do modelo de segunda ordem

\begin{tabular}{lccccc}
\hline \multicolumn{1}{c}{ Caminho } & $\begin{array}{c}\text { Beta } \\
\text { (standard } \\
\text { loadings) }\end{array}$ & $\begin{array}{c}\mathbf{R}^{\mathbf{2}} \\
\text { (standard loadings } \\
\text { squared) }\end{array}$ & $\begin{array}{c}\text { Error } \\
\text { Variance }\end{array}$ & T & Sig. \\
\hline MUIPC $\rightarrow$ VP & 0,890 & 0,793 & 0,208 & 59,054 & \\
MUIPC $\rightarrow$ IP & 0,863 & 0,744 & 0,255 & 43,372 & $\mathrm{P}<0,001$ \\
MUIPC $\rightarrow$ US & 0,905 & 0,82 & 0,181 & 67,65 & \\
Total Loadings & 2,658 & 2,356 & 0,644 & - & - \\
Total Loadings Squared & 7,065 & 5,550 & - & - & \\
Total Loadings / Number of factors & AVE & 0,886 & & & \\
Total Loadings Squared / (Total Errors & CR & 0,916 & & & \\
of Variance + Total Loading Squared) & & & & &
\end{tabular}

Nota: número de fatores $=4$

Apêndice E - Estatísticas descritivas das variáveis

\begin{tabular}{ccc}
\hline Variável & Média & Desvio Padrão \\
\hline VP2 & 5,18 & 1,688 \\
VP3 & 5,11 & 1,728 \\
IP1 & 4,80 & 1,693 \\
IP2 & 5,29 & 1,594 \\
IP3 & 5,13 & 1,591 \\
US1 & 5,65 & 1,504 \\
US2 & 5,57 & 1,560 \\
US3 & 5,76 & 1,501 \\
\hline
\end{tabular}

$n=309$

Apêndice F - Estratificação da Amostra

\begin{tabular}{ccc}
\hline Gênero & Frequência & Percentual \\
\hline $\mathrm{F}$ & 124 & $40,1 \%$ \\
$\mathrm{M}$ & 185 & $59,9 \%$ \\
\hline Idade & & \\
\hline 18 a 30 & 173 & $56,0 \%$ \\
31 a 50 & 145 & $46,9 \%$ \\
mais de 51 & 9 & $2,9 \%$ \\
\hline
\end{tabular}

$n=309$ 